Andy Petros

Nia Taylor

Hendrick K. van Saene

Luciano Silvestri

\section{Gut overgrowth harms the critically ill}

Accepted: 16 March 2011

Published online: 28 June 2011

(C) Copyright jointly held by Springer and ESICM 2011

An author's reply to this comment is available at: doi:10.1007/s00134011-2288-z.

Dear Editor,

We read with interest the randomised controlled trial (RCT) by Oudhuis et al. [1] on the impact of probiotics versus selective digestive decontamination (SDD) on infection and mortality.

The working hypothesis of the Maastricht group was that probiotics are as good as SDD in reducing morbidity and mortality in the critically ill requiring treatment on the intensive care unit (ICU). They believed that probiotics are even superior to SDD as they do not have the potential disadvantage of antibiotics in terms of antimicrobial resistance. Indeed, the concept of exposing vast numbers of critically ill patients to broad-spectrum multiple drug cocktails runs counter to existing theoretical models (and dogma) related to the genesis and promotion of antimicrobial resistance [2].

There was a tendency towards more infections in patients receiving probiotics compared with SDD: $31 \%$ in the probiotic group versus $24 \%$ in the SDD group (OR $1.68,95 \%$ CI $0.91-3.08 ; p=0.10)$. The additional antibiotic use measured by the total mean number of defined daily dose
(DDD)/100 patient days was significantly higher in the probiotic group than in the SDD group (141.7 vs. 108.7; $p=0.008)$. Among Pseudomonas aeruginosa isolates, resistance to ceftazidime ( $p=0.02$ ), ciprofloxacin $(p<0.001)$, piperacillin $(p=0.004)$ and piperacillin-tazobactam $(p=0.02)$ was significantly higher in the probiotic group than in the SDD group. The study was not powered to detect a difference in mortality. These results of the Maastricht RCT confirm a recent probiotic meta-analysis [3]. But what is more disturbing is the fact that the Dutch authors do not make any efforts to explain the failure of their RCT.

Gut overgrowth defined as $\geq 10^{5}$ potential pathogens per $\mathrm{ml}$ of digestive tract secretion has been

acknowledged to cause immunosuppression and generalised

inflammation [4]. Gut overgrowth is the crucial event preceding endogenous infections and is a risk factor for de novo development of antimicrobial resistance [5]. Although the Dutch authors did not measure overgrowth in surveillance cultures, it is highly likely that the ICU patients on probiotics had gut overgrowth promoting endogenous infections and antimicrobial resistance. SDD is a prophylaxis using antimicrobials designed to control overgrowth.

Parenteral cefotaxime eradicates gut overgrowth of 'normal' bacteria such as $S$. pneumoniae, $H$. influenzae and $S$. aureus in the throat and E. coli in the gut due to high salivary and biliary concentrations. Enteral polyenes eradicate gut overgrowth of 'normal' Candida species. Enteral polymyxin and tobramycin prevent or eradicate, if already present, gut overgrowth of 'abnormal' aerobic gram-negative bacilli (AGNB). Finally, enteral vancomycin controls gut overgrowth of 'abnormal' methicillin-resistant Staphylococcus aureus (MRSA). All SDD-RCTs in which overgrowth was measured invariably show eradication of gut overgrowth [4].

In conclusion, we feel sorry for the Maastricht group. Their intention was not to show superiority of SDD over probiotics, but, their results are in line with the vast evidence base of SDD.

\section{References}

1. Oudhuis GJ, Bergmans DC, Dormans T, Zwaveling JH, Kessels A, Prins MH, Stobberingh EE, Verbon A (2011)

Probiotics versus antibiotic decontamination of the digestive tract: infection and mortality. Intensive Care Med 37:110-117. doi:

10.1007/s00134-010-2002-6

2. Laupland KB, Fisman DN (2009)

Selective digestive tract decontamination: a tough pill to swallow. Can J Infect Dis Med Microbiol 20:9-11

3. Silvestri L, van Saene HK, Gregori D, Agostini S, Francescon M, Taylor N (2010) Probiotics to prevent ventilatorassociated pneumonia: no robust evidence from randomised controlled trials. Crit Care Med 38:1616-1617

4. van Saene HKF, Damjanovic V, Murray AE, de la Cal MA (1996) How to classify infections in intensive care units. The carrier state, a criterion whose time has come? J Hosp Infect 33:1-12

5. van Saene HK, Taylor N, Damjanovic V, Sarginson RE (2008) Microbial gut overgrowth guarantees increased spontaneous mutation leading to polyclonality and antibiotic resistance in the critically ill. Curr Drug Targets 9:419-421

\section{A. Petros}

Paediatric and Neonatal Intensive Care Unit, Great Ormond Street Hospital for Children, London, UK

N. Taylor

Institute of Ageing and Chronic Disease, University of Liverpool, Liverpool, UK

H. K. van Saene (๘)

Institute of Ageing and Chronic Disease, University of Liverpool, Liverpool, UK e-mail: nia.taylor@liv.ac.uk

\section{Silvestri}

Unit of Anesthesia and Intensive Care, Department of Emergency, Presidio Ospedaliero, Gorizia, Italy 